\title{
3-Demethoxy-3-Glycosylaminothiocolchicines:
}

\section{Synthesis of a New Class of Putative Muscle Relaxant}

\section{Compounds}

Maria Luisa Gelmi, ${ }^{\$}$ Donato Pocar,${ }^{\$}$ Guido Pontremoli, ${ }^{\$}$ Sara Pellegrino, ${ }^{\$}$ Ezio Bombardelli, ${ }^{\ddagger}$ Gabriele Fontana, ${ }^{\ddagger}$ Antonella Riva, ${ }^{\ddagger}$ Walter Balduini, ${ }^{\dagger}$ Silvia Carloni, ${ }^{\dagger}$ Mauro Cimino, ${ }^{\dagger}$ Francis Johnson $^{\&}$

Istituto di Chimica Organica “A. Marchesini", Facoltà di Farmacia, Università di Milano, Via Venezian 21, I-20133 Milano, Italy. Indena S.p.a., Via Ortles 19, Milano, Italy. Istituto di Farmacologia e Farmacognosia, Facoltà di Farmacia, Università di Urbino “Carlo Bo”, Via S. Chiara 27, 61029 Urbino, Italy. Department of Chemistry, State University of New York at Stony Brook, Stony Brook, New York 11790-3400.

\section{Contents:}

1. Table TS1. Significant Chemical Shifts and $J$ Values for Anomers $\alpha-7$ and $\beta-7$. Pg. S2

2. General. Pg. S2

3. Analytical and spectroscopic data for compounds 7. Pg. S2-S5

4. Table TS2. Analytical data for compounds 3,5,7. Pg S6 
Table TS1. Significant Chemical Shifts and $J$ Values for Anomers $\alpha-7$ and $\beta-7$.

\begin{tabular}{|c|c|c|c|c|c|c|c|}
\hline Compd. & $\mathrm{H}-4$ & NH-3 $(J$ Hz) & $\mathrm{H}^{\prime}(J \mathrm{~Hz})$ & Compd. & $\mathrm{H}-4$ & NH-3 $(J \mathrm{~Hz})$ & $\mathrm{H}^{\prime}(J \mathrm{~Hz})$ \\
\hline$\alpha-7 \mathbf{a}$ & 6.76 & $5.58(3.8)$ & $4.91(5.0,3.8)$ & $\beta-7 a$ & 6.48 & $5.78(6.5)$ & $4.34(8.1,6.5)$ \\
\hline$\alpha-7 b$ & 6.49 & $6.01(9.8)$ & $4.93(9.8,2.0)$ & $\beta-7 \mathbf{b}$ & 6.45 & $6.21(8.3)$ & $4.61(8.3,4.8)$ \\
\hline$\alpha-7 c$ & 6.45 & $5.68(7.1)$ & $4.72(8.4,7.1)$ & $\beta-7 c$ & 6.48 & $6.13(8.7)$ & $4.90(8.7,2.7)$ \\
\hline$\alpha-7 d$ & 6.55 & $5.46(5.9)$ & $4.88(5.9,3.8)$ & $\beta-7 d$ & 6.46 & $5.81(7.2)$ & $4.41(7.8,7.2)$ \\
\hline \multirow[t]{2}{*}{$\alpha-7 e$} & 6.49 & $5.64(9.9)$ & $4.84(9.9,10.7)$ & $\beta-7 f$ & 6.48 & $5.72(10)$ & $4.82(10.0)$ \\
\hline & & & & $\beta-7 g$ & 6.46 & $5.65(6.8)$ & $4.36(8.6,6.8)$ \\
\hline
\end{tabular}

General. Mps were determined using a Büchi 510 (capillary) apparatus. ${ }^{1} \mathrm{H}$ NMR spectra were recorded with an AVANCE 500 Bruker at $500 \mathrm{MHz}$ for ${ }^{1} \mathrm{H}$ NMR and $100 \mathrm{MHz}$ for ${ }^{13} \mathrm{C}$ NMR. Chemical shifts, relative to TMS as internal standard, are given in $\delta$ values. $J$ are given in Hz. TLC: ready-to-use silica gel plates. Column chromatography: silica gel [Kieselgel 60-70 230 ASTM (Merck)] with the eluant indicated. IR spectra were taken with a Perkin-Elmer 1725X FT-IR spectrophotometer. $[\alpha]_{D}^{25}$ were measured with a Perkin Elmer MODEL343 Plus Polarimeter.

\section{Analytical and spectroscopic data for compounds 7}


3-Demethoxy-3D-glucopyranosylaminothiocolchicine 7a: IR (Nujol) $v_{\max } 1667,1620 \mathrm{~cm}^{-1} ; \boldsymbol{\alpha - 7 a :}{ }^{1} \mathrm{H}$ NMR (DMSO) $\delta 8.63$ (d, $J=7.6 \mathrm{~Hz}, 1 \mathrm{H}$, exch), 7.25, 7.18 (AB system, $J=10.3 \mathrm{~Hz}, 2 \mathrm{H}), 7.01$ (s, $1 \mathrm{H}$ ), 6.76 (s, 1H), 5.58 (d, $J=3.8 \mathrm{~Hz}, 1 \mathrm{H}$, exch), 5.36 (bs, 1H, exch), 5.22 (bs, 1H, exch), 4.91 (dd, $J=5.0$, 3.8 Hz, 1H), 4.41-4.35 (m, 1H), 3.83 (s, 3H), 3.73-3.64 (m, 1H), 3.64-3.57 (m, 1H), 3.51 (s, 3H), 3.503.40 (m, 1H), 3.19-3.10 (m, 2H), 2.55-2.45 (m, 1H), 2.41 (s, 3H), 2.23-2.15 (m, 1H), 2.07-1.95 (m, 1H), 1.87-1.78 (m, 1H), 1.81 (s, 3H); ${ }^{13} \mathrm{C}$ NMR (DMSO) $\delta 181.6,169.0,157.0,152.0,150.1,141.7,138.9$, 138.6, 135.2, 134.2, 128.4, 127.2, 122.3, 108.1, 85.5, 78.1, 77.9, 73.3, 70.8, 61.5, 60.9, 60.9, 51.9, 36.3, 29.9, 22.9, 14.8. $\beta$-7a: ${ }^{1} \mathrm{H}$ NMR (DMSO) $\delta 8.63$ (d, $J=7.6 \mathrm{~Hz}, 1 \mathrm{H}$, exch), 7.26, 7.15 (AB system, $J=$ $10.3 \mathrm{~Hz}, 2 \mathrm{H}), 7.01$ (s, 1H), 6.48 (s, 1H), 5.78 (d, $J=6.5 \mathrm{~Hz}, 1 \mathrm{H}$, exch), 5.19 (d, $J=10.2 \mathrm{~Hz}, 1 \mathrm{H}$, exch), 5.18 (d, $J=4.6 \mathrm{~Hz}, 1 \mathrm{H}$, exch), 5.04 (d, $J=3.8 \mathrm{~Hz}, 1 \mathrm{H}$, exch), 4.41-4.35 (m, 1H), 4.34 (dd, $J=8.1,6.5$ Hz, 1H), 3.79 (s, 3H), 3.73-3.64 (m, 1H), 3.64-3.57 (m, 1H), 3.52 (s, 3H), 3.50-3.40 (m, 1H), 3.32-3.23 (m, $2 \mathrm{H}), 2.55-2.45$ (m, 1H), 2.41 (s, 3H), 2.23-2.15 (m, 1H), 2.07-1.95 (m, 1H), 1.87-1.78 (m, $1 \mathrm{H}), 1.81$ (s, 3H); ${ }^{13} \mathrm{C}$ NMR (DMSO) 181.6, 169.0, 157.0, 152.0, 150.1, 141.7, 138.9, 138.6, 135.2, 134.2, 128.4, 127.2, 122.3, 108.1, 85.5, 78.1, 77.9, 73.3, 70.8, 61.5, 60.9, 60.9, 51.9, 36.3, 29.9, 23.3, 14.8 .

3-D-Arabinopyranosylamino-3-demethoxy-thiocolchicine 7b: IR (Nujol) $v_{\max } 1667,1620 \mathrm{~cm}^{-1}$; $\boldsymbol{\alpha}$ 7b: ${ }^{1} \mathrm{H}$ NMR (DMSO) $\delta 8.59$ (d, $J=8.7 \mathrm{~Hz}, 1 \mathrm{H}$ exch), 7.25, 7.18 (AB system, $J=10.4 \mathrm{~Hz}, 2 \mathrm{H}$ ), 7.01 (s, 1H), 6.49 (s, 1H), 6.01 (d, $J=9.8 \mathrm{~Hz}, 1 \mathrm{H}$ exch), 5.77 (d, $J=6.1 \mathrm{~Hz}, 1 \mathrm{H}$ exch), 5.48 (d, $J=4.2 \mathrm{~Hz}$, 1H exch), 5.29 (d, $J=4.4 \mathrm{~Hz}, 1 \mathrm{H}$ exch), 4.93 (dd, $J=9.8,2.0 \mathrm{~Hz}, 1 \mathrm{H}), 4.41-4.32$ (m, 1H), 3.78 (m, 3H), 3.68-3.64 (m, 1H), 3.62-3.57 (m, 3H), 3.52 (s, 3H), 3.46-3.37 (m, 1H), 2.55-2.48 (m, 1H), 2.41 (s, 3H), 2.21-2.16 (m, 1H), 2.05-1.98 (m, 1H), 1.86-1.78 (m, 1H), 1.85 (s, 3H); ${ }^{13} \mathrm{C}$ NMR (DMSO) $\delta ~ 181.6$, 168.9, 157.2, 152.2, 150.4, 141.9, 141.0, 138.7, 135.4, 134.2, 128.3, 127.2, 122.4, 107.4, 81.6, 73.5, 70.5, 68.2, 65.7, 61.1, 60.9, 51.9, 36.3, 29.9, 23.3, 22.7, 14.8. $\beta$-7b: ${ }^{1} \mathrm{H}$ NMR (DMSO) $\delta 8.56$ (d, $J=7.7$ Hz, 1H exch), 7.26, 7.18 (AB system, $J=10.5$ Hz, 2H), 7.01 (s, 1H), 6.45 (s, 1H), 6.21 (d, $J=8.3 \mathrm{~Hz}$, 1H exch), 5.57 (d, $J=9.2 \mathrm{~Hz}, 1 \mathrm{H}$ exch), 5.51 (d, $J=5.6 \mathrm{~Hz}, 1 \mathrm{H}$ exch), 4.61 (dd, $J=8.3,4.8 \mathrm{~Hz}, 1 \mathrm{H}$ ), 4.57 (d, $J=6.1 \mathrm{~Hz}, 1 \mathrm{H}$ exch), 4.41-4.32 (m, 1H), 3.80 (s, 3H), 3.68-3.64 (m, 1H), 3.62-3.57 (m, 3H), 
3.52 (s, 3H), 3.46-3.37 (m, 1H), 2.55-2.48 (m, 1H), 2.41 (s, 3H), 2.21-2.16 (m, 1H), 2.05-1.98 (m, 1H),

1.86-1.78 (m, 1H), 1.84 (s, 3H); ${ }^{13} \mathrm{C}$ NMR (DMSO) $\delta$ 181.6, 168.9, 157.2, 152.2, 150.4, 141.9, 141.0, 138.7, 135.4, 134.2, 128.3, 127.2, 122.4, 107.4, 81.6, 73.5, 70.5, 68.2, 65.7, 61.1, 60.9, 51.9, 36.3, 29.9, 23.3, 22.7, 14.8 .

3-Demethoxy-3-D-lyxopyranosylaminothiocolchicine 7c: IR (Nujol) $v_{\max } 1667,1620 \mathrm{~cm}^{-1} ; \boldsymbol{\alpha - 7 c :}{ }^{1} \mathrm{H}$ NMR (DMSO) $\delta 8.58$ (d, $J=7.6 \mathrm{~Hz}, 1 \mathrm{H}$, exch), 7.26, 7.17 (AB system, $J=10.7 \mathrm{~Hz}, 2 \mathrm{H}$ ), 7.03 (s, 1H), 6.45 (s, 1H), 5.68 (d, $J=7.1 \mathrm{~Hz}, 1 \mathrm{H}$ exch), 5.18 (d, $J=4.5 \mathrm{~Hz}, 1 \mathrm{H}$ exch), 4.95 (d, $J=3.9 \mathrm{~Hz}, 1 \mathrm{H}$ exch), 4.83 (d, $J=7.1 \mathrm{~Hz}, 1 \mathrm{H}$ exch), 4.72 (dd, $J=8.4,7.1 \mathrm{~Hz}, 1 \mathrm{H}$ ), 4.40-4.36 (m, 1H), 3.81 (s, 3H), 3.75-3.72 (m, 2H), 3.52 (s, 3H), 3.55-3.51 (m, 1H), 3.20-3.16 (m, 1H), 3.18-3.15 (m, 1H), 2.56-2.48 (m, 1H), 2.41 (s, 3H), 2.24-2.15(m, 1H), 2.07-1.95 (m, 2H), 1.86-1.78 (m, 1H), 1.84 (s, 3H); ${ }^{13} \mathrm{C}$ NMR (DMSO) $\delta$ 181.6, 168.9, 157.1, 151.9, 150.2, 141.7, 141.0, 138.7, 135.2, 134.2, 128.4, 127.2, 122.1, 106.4, 82.1, 71.2, 70.1, 67.8, 63.6, 60.8, 60.7, 51.9, 36.3, 29.9, 23.3, 22.9, 14.8. $\beta$-7c: ${ }^{1} \mathrm{H}$ NMR (DMSO) $\delta 8.59$ (d, $J$ $=7.3 \mathrm{~Hz}, 1 \mathrm{H}$, exch), 7.25, 7.15 (AB system, $J=10.4 \mathrm{~Hz}, 2 \mathrm{H}), 7.03(\mathrm{~s}, 1 \mathrm{H}), 6.48$ (s, 1H), 6.13 (d, $J=8.7$ Hz, 1H, exch), 5.06 (d, $J=5.5 \mathrm{~Hz}, 1 \mathrm{H}$ exch), 4.90 (dd, $J=8.7,2.7 \mathrm{~Hz}, 1 \mathrm{H}), 4.86$ (d, $J=4.5 \mathrm{~Hz}, 1 \mathrm{H}$ exch), 4.40-4.36 (m, 1H), 3.82-3.79 (m, 2H), 3.79 (s, 3H), 3.62-3.58 (m, 1H), 3.52 (s, 3H), 3.55-3.51 (m, 1H), 3.49-3.46 (m, 1H), 3.18-3.15 (m, 1H), 2.56-2.48 (m, 1H), 2.41 (s, 3H), 2.24-2.15(m, 1H), 2.071.95 (m, 2H), 1.86-1.78 (m, 1H), 1.84 (s, 3H); ${ }^{13} \mathrm{C}$ NMR (DMSO) $\delta$ 181.6, 169.0, 157.1, 152.0, 150.3, 141.7, 141.0, 138.9, 135.2, 134.3, 128.4, 127.2, 122.2, 107.4, 81.6, 73.6, 71.5, 68.0, 65.5, 61.0, 60.9, 51.9, 36.2, 29.8, 23.3, 22.9, 14.8 .

3-Demethoxy-3-D-xylopyranosylaminothiocolchicine 7d: IR (Nujol) $v_{\max } 1667,1620 \mathrm{~cm}^{-1}$; $\boldsymbol{\alpha - 7 d}$ : ${ }^{1} \mathrm{H}$ NMR (DMSO) $\delta$ 8.65-8.56 (m, 1H exch), 7.25, 7.17 (AB system, $J=10.6 \mathrm{~Hz}, 2 \mathrm{H}$ ), $7.02(\mathrm{~s}, 1 \mathrm{H}$ ), 6.55 (s, 1H), 5.46 (d, $J=5.9 \mathrm{~Hz}, 1 \mathrm{H}$ exch), 5.32 (d, $J=5.6 \mathrm{~Hz}, 1 \mathrm{H}$ exch), 5.21 (bs, $1 \mathrm{H}$ exch), 5.05 (d, $J$ $=5.4 \mathrm{~Hz}, 1 \mathrm{H}$ exch), 4.88 (dd, $J=5.9,3.8 \mathrm{~Hz}, 1 \mathrm{H}), 4.42-4.35$ (m, 1H), $3.82(\mathrm{~s}, 3 \mathrm{H}), 3.70-3.50(\mathrm{~m}, 2 \mathrm{H})$, 3.51 (s, 3H), 3.40-3.30 (m, 1H), 3.30-3.20 (m, 2H), 2.57-2.50 (m, 1H), 2.50 (s, 3H), 2.21-2.12 (m, 1H), 2.07-1.97 (m, 1H), 1.86-1.78 (m, 1H), 1.85 (s, 3H ); ${ }^{13} \mathrm{C}$ NMR (DMSO) $\delta 181.6,168.9,157.2,151.8$, 
150.1, 141.5, 138.8, 135.4, 134.4, 128.3, 127.2, 123.1, 108.0, 81.7, 77.2, 71.1, 67.8, 66.1, 60.9, 51.9, 36.3, 29.8, 23.3, 14.8. $\beta$-7d: ${ }^{1} \mathrm{H}$ NMR (DMSO) $\delta$ 8.65-8.56 (m, $1 \mathrm{H}$ exch), 7.25, 7.17 (AB system, $J=$ $10.6 \mathrm{~Hz}, 2 \mathrm{H}), 7.02$ (s, 1H), 6.46 (s, 1H), 5.81 (d, $J=7.2 \mathrm{~Hz}, 1 \mathrm{H}$ exch), 5.52 (t, $J=5.3 \mathrm{~Hz}, 1 \mathrm{H}$, exch), 5.11 (bs, 2H exch), 4.99 (d, $J=4.8 \mathrm{~Hz}, 1 \mathrm{H}$ exch), 4.42-4.35 (m, 1H), 4.41 (dd, $J=7.8,7.2 \mathrm{~Hz}, 1 \mathrm{H}$ ), 3.79 (s, 3H), 3.70-3.50 (m, 2H), 3.49 (s, 3H), 3.40-3.30 (m, 1H), 3.30-3.20 (m, 2H), 2.57-2.50 (m, 1H), 2.50 (s, 3H), 2.21-2.12 (m, 1H), 2.07-1.97 (m, 1H), 1.86-1.78 (m, 1H), 1.84 (s, 3H); ${ }^{13} \mathrm{C}$ NMR (DMSO) $\delta$ 181.6, 168.9, 157.1, 151.9, 150.2, 141.4, 138.7, 135.2, 134.2, 128.3, 127.2, 122.3, 107.5, 85.7, 77.4, 72.9, 70.2, 66.6, 61.1, 61.0, 51.9, 36.3, 29.8, 22.9, 14.8.

3-Demethoxy-3-L-rhamnopyranosylaminothiocolchicina $\alpha$-7e: mp $185-188{ }^{\circ} \mathrm{C} ;[\alpha]_{25} \mathrm{D}-299^{\circ}(\mathrm{c}$ 0.4, MeOH); IR (Nujol) $v_{\max } 1667,1620 \mathrm{~cm}^{-1} ;{ }^{1} \mathrm{H}-\mathrm{NMR}$ (DMSO) $\delta 8.58$ (d, $J=7.3 \mathrm{~Hz}, 1 \mathrm{H}$ exch.), 7.27, 7.14 (AB system, $J=10.5 \mathrm{~Hz}, 2 \mathrm{H}$ ), 7.02 (s, 1H), 6.49 (s, 1H), 5.64 (d, $J=9.9 \mathrm{~Hz}, 1 \mathrm{H}$ exch), 5.12 (d, $J=$ 4.4 Hz, 1H exch.), 4.84 (dd, $J=10.7,9.9$ Hz, 1H), 4.79 (brs, 2H exch), 4.41-4.38 (m, 1H), 3.80 (s, 3H), 3.79-3.78 (m, 1H), 3.51 (s, 3H), 3.45-3.15 (m, 3H), 2.55-2.47 (m, 1H), 2.40 (s, 3H), 2.22-2.17 (m, 1H), 2.06-1.97 (m, 1H). 1.85 (s, 3H), 1.84-1.81 (m, 1H), 1.11 (d, $J=5.6 \mathrm{~Hz}, 3 \mathrm{H}) ;{ }^{13} \mathrm{C}$ NMR (DMSO) $\delta$ 182.0, 169.4, 157.5, 152.3, 150.6, 140.8, 139.0, 135.6, 134.6, 128.7, 127.6, 122.6, 108.1, 94.9, 80.9, 74.9, 73.4, 72.7, 71.9, 61.4, 61.2, 36.7, 29.9, 23.3, 22.9, 18.9 .

3-Demethoxy-3-D-Mannopyranosylaminothiocolchicine $\beta$-7f: (mixture with $10 \%$ of its conformer $\beta-7^{\prime}$ f). Mp $165-167^{\circ} \mathrm{C} ;[\alpha]_{25}{ }^{\mathrm{D}}-395^{\circ}$ (c 0.44, MeOH); IR (Nujol) $v_{\max } 1667,1620 \mathrm{~cm}^{-1} ; \beta-7 f:{ }^{1} \mathrm{H}$ NMR (DMSO) $\delta 8.59$ (d, $J=7.5 \mathrm{~Hz}, 1 \mathrm{H}$, exch.), 7.25, 7.16 (AB system, $J=10.3 \mathrm{~Hz}, 2 \mathrm{H}$ ), 7.01 (s, 1H), 6.48 (s, 1H), 5.72 (d, $J=10.0 \mathrm{~Hz}, 1 \mathrm{H}$, exch), 5.13 (d, $J=5.2 \mathrm{~Hz}, 1 \mathrm{H}$, exch), 4.80 (d, $J=5.4 \mathrm{~Hz}, 1 \mathrm{H}$, exch), 4.82 (d, $J=10 \mathrm{~Hz}, 1 \mathrm{H}), 4.76$ (d, $J=5.0 \mathrm{~Hz}, 1 \mathrm{H}$, exch), 4.43 (t, $J=6.1 \mathrm{~Hz}, 1 \mathrm{H}$, exch), 4.42-4.33 (m, 1H), 3.49 (s, 3H), 3.83 (s, 3H), 3.77-3.52 (m, 3H), 3.49-3.35 (m, 2H), 3.29-3.15 (m, 1H), 2.52-2.45 (m, 1H), 2.42 (s, 3H), 2.20-2.15 (m, 1H), 2.05-1.98 (m, 1H), 1.84-1.78 (m, 1H), 1.86 (s, 3H); ${ }^{13} \mathrm{C}$ NMR (DMSO) $\delta$ 181.6, 169.0, 157.1, 152.0, 150.1, 140.4, 138.8, 138.6, 135.2, 134.3, 128.4, 127.2, 122.3, 108.2, 94.5, 78.5, 74.9, 71.3, 67.9, 67.6, 61.6, 60.9, 60.8, 51.9, 36.2, 29.9, 23.3, 14.8; $\beta-7$ 'f, 
significative signals: ${ }^{1} \mathrm{H}$ NMR (500 MHz, DMSO): 6.72 (s, 1H), 5.61 (d, $J=4.1 \mathrm{~Hz}, 1 \mathrm{H}$, exch), 4.88 (d, $J=4.1 \mathrm{~Hz}, 1 \mathrm{H}), 4.60$ (d, $J=5.5 \mathrm{~Hz}, 1 \mathrm{H}$, exch), 4.48 (d, $J=5.3 \mathrm{~Hz}, 1 \mathrm{H}$, exch), 4.30 (t, $J=5.8 \mathrm{~Hz}, 1 \mathrm{H}$, exch); ${ }^{13} \mathrm{C}$ NMR (500 MHz, DMSO) $\delta: 181.6,169.0,157.1,152.0,150.1,140.4,138.8,138.6,135.2$, 134.3, 128.4, 127.2, 122.3, 108.2, 80.8, 73.6, 71.9, 71.1, 67.9, 62.0, 61.6, 60.9, 60.8, 51.9, 36.2, 29.9, 23.3, 22.9, 14.8 .

3-Demethoxy-3-L-fucopyranosylaminothiocolchicine $\beta$-7g: mp $185-188{ }^{\circ} \mathrm{C} ;[\alpha]_{25}{ }^{\mathrm{D}}-299^{\circ}(\mathrm{c} \quad 0.4$, MeOH); IR (Nujol) $v_{\max } 1667,1620 \mathrm{~cm}^{-1}$; ${ }^{1} \mathrm{H}-\mathrm{NMR}$ (DMSO) $\delta 8.60$ (d, $J=7.3 \mathrm{~Hz}, 1 \mathrm{H}$ exch), 7.26, 7.17 (AB system, $J=10.3 \mathrm{~Hz}, 2 \mathrm{H}$ ), 7.02 (s, 1H), 6.46 (s, 1H), 5.65 (d, $J=6.8 \mathrm{~Hz}, 1 \mathrm{H}$ exch), 4.94 (d, $J=6.6$ Hz, 1H exch), 4.74 (d, $J=5.5 \mathrm{~Hz}, 1 \mathrm{H}$ exch), 4.40 (d, $J=5.1 \mathrm{~Hz}, 1 \mathrm{H}$ exch), 4.40-4.35 (m, 1H), 4.36 (dd, $J=8.6,6.8 \mathrm{~Hz}, 1 \mathrm{H}), 3.81$ (s, 3H), 3.75-3.65 (m, 1H), 3.70-3.38 (m, 5H), 3.65-3.50 (m, 2H), 3.51 (s, 3H), 3.50-3.40 (m, 1H), 2.55-2.47 (m, 1H), 2.40 (s, 3H), 2.22-2.17 (m, 1H), 2.06-1.97 (m, 1H), 1.88$1.78(\mathrm{~m}, 1 \mathrm{H}), 1.84$ (s, 3H), $1.10(\mathrm{~d}, J=6.1 \mathrm{~Hz}, 3 \mathrm{H}) ;{ }^{13} \mathrm{C}$ NMR (DMSO) $\delta 181.6,168.9,157.0,152.0$, 150.2, 141.7, 138.7, 138.6, 135.2, 134.2, 128.4, 127.2, 122.1, 107.4, 85.4, 74.6, 71.9, 70.9, 70.1, 60.9, 60.9, 51.9, 36.3, 29.9, 23.3, 17.3, 14.8 . 
Table TS2. Analytical data for compounds 3,5,7.

\begin{tabular}{|c|c|c|c|c|c|c|c|c|}
\hline \multirow[t]{2}{*}{ Compd. } & \multirow{2}{*}{$\begin{array}{c}\mathrm{MS} \mathrm{m} / \mathrm{z} \\
\left(\mathrm{M}^{+}\right)\end{array}$} & \multirow{2}{*}{$\begin{array}{l}\text { Molecular } \\
\text { Formula }\end{array}$} & \multicolumn{3}{|c|}{ Calcd. } & \multicolumn{3}{|c|}{ Found } \\
\hline & & & $\mathrm{C}$ & $\mathrm{H}$ & $\mathrm{N}$ & $\mathrm{C}$ & $\mathrm{H}$ & $\mathrm{N}$ \\
\hline 3 & 533 & $\mathrm{C}_{22} \mathrm{H}_{22} \mathrm{~F}_{3} \mathrm{NO}_{7} \mathrm{~S}_{2}$ & 49.52 & 4.16 & 2.63 & 49.33 & 4.22 & 2.51 \\
\hline 5 & 400.1 & $\mathrm{C}_{21} \mathrm{H}_{24} \mathrm{~N}_{2} \mathrm{O}_{4} \mathrm{~S}$ & 62.98 & 6.04 & 6.99 & 62.84 & 6.08 & 6.89 \\
\hline $7 a$ & 563.2 & $\mathrm{C}_{27} \mathrm{H}_{34} \mathrm{~N}_{2} \mathrm{O}_{9} \mathrm{~S}$ & 57.64 & 6.09 & 4.98 & 57.32 & 6.44 & 4.54 \\
\hline $7 \mathbf{b}$ & 533.3 & $\mathrm{C}_{26} \mathrm{H}_{32} \mathrm{~N}_{2} \mathrm{O}_{8} \mathrm{~S}$ & 58.63 & 6.06 & 5.26 & 58.33 & 6.55 & 4.94 \\
\hline $7 c$ & 533.3 & $\mathrm{C}_{26} \mathrm{H}_{32} \mathrm{~N}_{2} \mathrm{O}_{8} \mathrm{~S}$ & 58.63 & 6.06 & 5.26 & 58.29 & 6.52 & 4.98 \\
\hline $7 d$ & 533.3 & $\mathrm{C}_{26} \mathrm{H}_{32} \mathrm{~N}_{2} \mathrm{O}_{8} \mathrm{~S}$ & 58.63 & 6.06 & 5.26 & 58.28 & 6.65 & 4.95 \\
\hline $7 e$ & 547.2 & $\mathrm{C}_{27} \mathrm{H}_{34} \mathrm{~N}_{2} \mathrm{O}_{8} \mathrm{~S}$ & 59.32 & 6.27 & 5.12 & 58.98 & 6.55 & 4.99 \\
\hline $7 f$ & 562 & $\mathrm{C}_{27} \mathrm{H}_{34} \mathrm{~N}_{2} \mathrm{O}_{9} \mathrm{~S}$ & 57.64 & 6.09 & 4.98 & 57.33 & 6.35 & 4.49 \\
\hline $7 g$ & 547.2 & $\mathrm{C}_{27} \mathrm{H}_{34} \mathrm{~N}_{2} \mathrm{O}_{8} \mathrm{~S}$ & 59.32 & 6.27 & 5.12 & 58.88 & 6.50 & 4.99 \\
\hline
\end{tabular}

